\title{
RELATION BETWEEN THE LONGITUDINAL FIELD AND THE RADIAL VELOCITY IN SUNSPOTS
}

\author{
A. A. PEVTSOV \\ Institute of Solar-Terrestrial Physics, \\ Irkutsk 33, P.O.Box 4026, 664033, Russia
}

\begin{abstract}
We describe some new aspects of the observed relationship between the longitudinal magnetic field $\left(B_{\text {long }}\right)$ and the Doppler velocity $\left(V_{D}\right)$ and propose an explanation of the effect in terms of a two-component penumbra model.
\end{abstract}

\section{OBSERVATIONS}

We used 49 magnetograms taken at the vector-magnetograph of the Sayan Observatory (Grigoryev et al.,1985) for 14 regular sunspots. The observations were made in the line Fe I $525.022 \mathrm{~nm}$, with spatial resolutions of $2 \times 2$ " and $4 \times 4$ ". Line-of-sight velocities were measured by the magnetograph Doppler compensator simultaneously with the observation of magnetic fields.

A direct comparison of the magnetic field and Doppler velocity maps shows both a correlation between $\mathrm{B}_{\text {long }}$ and $\mathrm{V}_{D}$, and the non-coincidence of their neutral lines and hills.For some sunspots, the largest Doppler velocities were observed on the neutral line of the longitudinal field, that is, in that place of the penumbra where the magnetic field vector is perpendicular to the line of sight (For example, see Pevtsov, 1992). We assumed that the observed picture is associated with averaging, by the magnetograph aperture, fine-structure elements of penumbra.

\section{A TWO-COMPONENT PENUMBRA MODEL AND THE RELATIONSHIP} BETWEEN B Bong AND $V_{0}$

The spectral line profile was considered to be the superposition of profiles from bright (BR) penumbra elements with a non-horizontal magnetic field and dark (DR) elements with a horizontal magnetic field. The field strength in DR is by $200-400 \mathrm{G}$ higher as compared with $\mathrm{BR}$. In DR material moves out of the sunspot with a maximum velocity of $4 \mathrm{~km} / \mathrm{s}$, and in BR material moves down into the sunspot with a maximum velocity of $1 \mathrm{~km} / \mathrm{s}$. (For references, see Pevtsov (1988). After calculating the averaged spectral line profile, values of 
Doppler velocity and magnetic field were calculated to be obtained for such a profile in observations with our magnetograph.
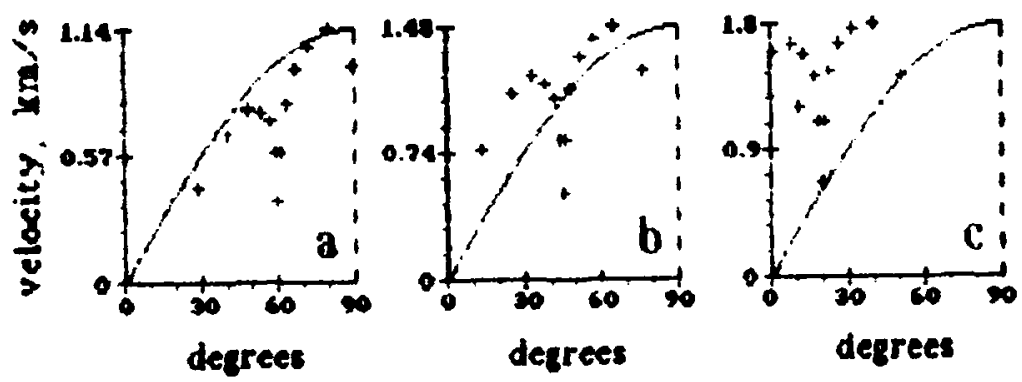

Fig 1. The dependence of the Doppler velocity on the modulus of slope angle $g$ of the magnetic field vector to the line of sight for a twocomponent penumbra model (solid line $-V_{\max } \times \operatorname{SIN}(|\gamma|)$ ). $a-\theta=30, \mathrm{~b}-$ $\theta=45, \mathrm{c}-\theta=70$.

Figure 1 presents results of our calculations. For $\theta=30$ (Fig.1a), the dependence corresponds quite well to the orthogonality (or anti-correlation) of the field and the velocity; with increasing $\theta$ (Fig.1b, $\theta=45$ ), this apparent orthogonality is no longer valid, and for large angular distances ( $\theta=70$, Fig. 1c) it is superseded by parallelism (correlation) of the field and the velocity.

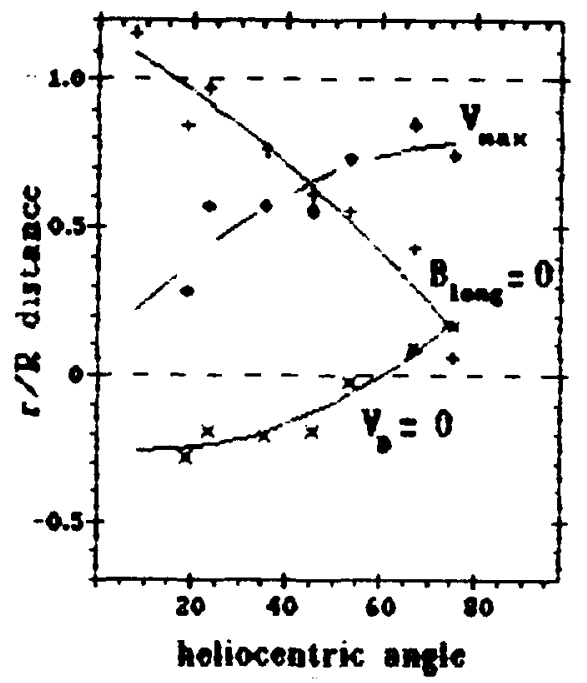

Fig. 2. Variations of positions of the neutral lines of the $\mathrm{V}_{D}$, the $\mathrm{B}_{\text {long }}$ and the maximum red shift $V_{D}$ at different angular positions of sunspots.

Let us now consider how the observed field and velocity correlation varies, depending on the angular sunspot position. Figure 2 shows the averaged dependences of the positions of the $\mathrm{V}_{D}=\mathrm{O}$ and $\mathrm{B}_{\text {long }}=\mathrm{O}$ lines and the maximum red shift velocity $\left(V_{D}\right)$ with respect to the sunspot center. Solid 
lines represent a parabolic approximation of these values. Near the disk center, the maximum red shift velocity lies near the neutral line of the longitudinal field but not exactly on it. A maximum anti-correlation is observed with the angular position of sunspots $(\theta)$ of about $45 \mathrm{deg}$. Near the limb, the neutral lines of the longitudinal field and the Doppler velocity coincide.

\section{CONCLUSION}

A different correlation between the longitudinal magnetic field and the Doppler velocity is observed at different angular positions of sunspots. Near the disk center, there is an anti-correlation of the field and the velocity when the maximum red shift velocity in the Evershed effect corresponds exactly to the neutral line of the longitudinal field. Near the limb, the neutral lines of the field and velocity coincide. A possible explanation would be that elements with a different direction of the field and the velocity exist in the sunspot penumbra. As our numerical calculations show, the averaging of such fine structure by the magnetograph aperture can lead to the above effects.

\section{ACKNOWLEDGEMENTS}

Part of this work has been accomplished within the framework of the American Astronomical Society grant (1.9.6). I am grateful to the AAS for finance support of this work. I wish to thank the International Astronomical Union for providing me with a travel grant, and the Local Organizing Committee for covering my living expenses in Beijing.

\section{REFERENCES}

Grigoryev, V. M., Kobanov, N. I., Osak, B. F., Stepanov, V. E., Selivanov, V. L. 1985, in Proceedings of the workshop on Measurements of Solar Vector Magnetic Field, ed. M.J.Hagyard, NASA Conference Publication 2374, p.231.

Pevtsov, A. A. 1988, in Proceedings of the XIII Consultative Meeting on Solar Physics, ed. R.B.Teplitskaj, Novosibirsk, 2, p.105.

Pevtsov, A. A., 1992, Solar Physics, 14165. 